\title{
Torque Ripple Minimization of PMSM Using Parameter Optimization Based Iterative Learning Control
}

\author{
Changliang Xia ${ }^{\dagger}$, Weitao Deng*, Tingna Shi* and Yan Yan*
}

\begin{abstract}
In this paper, a parameter optimization based iterative learning control strategy is presented for permanent magnet synchronous motor control. This paper analyzes the mechanism of iterative learning control suppressing PMSM torque ripple and discusses the impact of controller parameters on steady-state and dynamic performance of the system. Based on the analysis, an optimization problem is constructed, and the expression of the optimal controller parameter is obtained to adjust the controller parameter online. Experimental research is carried out on a $5.2 \mathrm{~kW}$ PMSM. The results show that the parameter optimization based iterative learning control proposed in this paper achieves lower torque ripple during steady-state operation and short regulating time of dynamic response, thus satisfying the demands for both steady state and dynamic performance of the speed regulating system.
\end{abstract}

Keywords: Iterative learning control, Permanent magnet synchronous motor, Periodic disturbance, Parameter optimization, Torque ripple minimization

\section{Introduction}

Permanent magnet synchronous motors(PMSM) which possess the characteristics of high power density, efficiency, robustness and simple structure, have been widely used in various industrial applications. However, the existence of torque ripple deteriorates the performance of PMSM speed regulating system $[1,2]$.

The torque ripple of PMSM is caused by nonideal factors including rotor flux harmonics, cogging torque, current sampling error and dead-time effect of inverter, etc[3]. These factors act on current control loop, leading to periodic fluctuations in q-axis current and in torque, which furthermore result in speed ripple, as mechanical inertia has weak effect of filtering low frequency harmonics.

A large number of methods have been proposed for suppressing torque ripple of PMSM, which can be divided into two categories. Generally, the first category is from the respect of motor design [4-6]. Such methods increase the complexity of the motor design and manufacturing process. The second is from the respect of the design of control system, i.e. reducing the torque ripple by optimizing control strategies. In early papers, current profiling is commonly used, which belongs to open-loop method [7]. This method is based on adequate knowledgement of torque characteristic of the motor. Its implementation requires plenty of off-line test and calculation, and is dependent on the motor parameters and environment. To

$\dagger$ Corresponding Author: School of Electrical Engineering and Automation, Tianjin University, China. (motor@tju.edu.cn)

* School of Electrical Engineering and Automation, Tianjin University, China. (\{dwt870104, tnshi, yanyan\}@tju.edu.cn)

Received: April 4, 2015; Accepted: November 19, 2015 achieve better robustness, closed-loop methods are proposed for suppressing torque ripple. Among them, a common method is to obtain optimal current value for generating constant torque on the basis of the relationship between current and torque of the motor, and then accordingly suppress torque or speed ripple with proposed torque or speed control algorithm. In [8], the quantity relationship between each harmonic of torque and the relevant harmonic of current is analyzed, and then the optimal current is calculated according to the current fedback to the controller. In [9], the current and back EMF are modeled in multidimensional space, and the current value for generating constant torque with minimized ohmic losses are derived by utilizing artificial neural networks. The compensation method based on observers is also a frequently used closed-loop method for suppressing torque ripple. In [10] Luenberger observer is employed to observe the harmonic torque disturbances based on their state space representation, and then the observer results are used for torque compensation; in [11] a load torque observer is proposed and adopted to suppress torque ripple. As the input of the PMSM is the stator voltage, and a control algorithm of the motor is implemented eventually on the control of reference voltage of the motor, another type of closed-loop method is presented by including control signal in the reference voltage generation. In [12] a current control scheme with an adaptive internal model is proposed to compensate reference voltage of the motor, and robust current control as well as torque ripple minimization is achieved in the presence of uncertainties like motor parameter variation. In [13] variable-structure torque and d-axis current controllers are adopted, and the estimated dynamic back-EMF vector containing flux harmonic infor- 
mation is used to compensate the reference voltage.

Due to the periodicity characteristic of the torque ripple, and the fixed relationship between the period and the rotational speed of the motor, the iterative learning control (ILC) is introduced to permanent magnet synchronous motor vector control system, in order to acquire a smooth torque control through the learning ability of iterative learning control for periodic signals [14-16]. To minimize the torque ripple, ILC with different learning laws are proposed [17-19], and implemented in time domain and frequency domain respectively $[20,21]$. As torque ripple also contains non-periodic components, which can not be suppressed by ILC, causing accumulative effect of nonperiodic disturbances, a relaxation factor $\alpha$ has to be included in the learning law of ILC in practical applications. The value of the relaxation factor has a significant effect on both steady state and dynamic performance of the system. If the value is selected too small, it would weaken the suppression effect on torque ripple; while it is selected too large, it would influence the stability of the system and meanwhile decrease the dynamic performance.

In this paper, iterative learning control with relaxation factor $\alpha$ is adopted to suppress torque ripple of PMSM. According to the effect of the parameter $\alpha$ on the performance of PMSM control system, this paper proposes a parameter optimization based iterative learning control, online adjusting the controller parameter, the relaxation factor $\alpha$, to achieve the purpose of reducing the torque ripple, while speeding up the convergence rate and improving the dynamic performance of the system compared with ILC with fixed $\alpha$.

\section{Mathematical Model of PMSM}

Neglecting the magnetic saturation and hysteresis and eddy current loss, in the synchronous rotating coordinate system, the $d-q$ axes model of PMSM is given as

$$
\left\{\begin{array}{l}
u_{\mathrm{d}}=R i_{\mathrm{d}}+\mathrm{d} \psi_{\mathrm{d}} / \mathrm{d} t-p \omega_{\mathrm{r}} \psi_{\mathrm{q}} \\
u_{\mathrm{q}}=R i_{\mathrm{q}}+\mathrm{d} \psi_{\mathrm{q}} / \mathrm{d} t+p \omega_{\mathrm{r}} \psi_{\mathrm{d}}
\end{array}\right.
$$

and

$$
\left\{\begin{array}{l}
\psi_{\mathrm{d}}=L_{\mathrm{d}} i_{\mathrm{d}}+\psi_{\mathrm{f}} \\
\psi_{\mathrm{q}}=L_{\mathrm{q}} i_{\mathrm{q}}
\end{array}\right.
$$

where, $u_{\mathrm{d}}, u_{\mathrm{q}}, i_{\mathrm{d}}$ and $i_{\mathrm{q}}$ are the $\mathrm{d}$, q-axes stator voltages and stator currents respectively; $R, L_{\mathrm{d}}$ and $L_{\mathrm{q}}$ are the stator resistance and the $\mathrm{d}$, q-axes equivalent inductances respectively; $\omega_{\mathrm{r}}$ is the rotor angular velocity; $\psi_{\mathrm{f}}$ is the permanent magnet flux; $p$ is the number of pole pairs.

The motor torque is given by

$$
T_{\mathrm{e}}=3 p\left(\psi_{\mathrm{d}} i_{\mathrm{q}}-\psi_{\mathrm{q}} i_{\mathrm{d}}\right) / 2
$$

The equation of the motor dynamics is

$$
J \mathrm{~d} \omega_{\mathrm{r}} / \mathrm{d} t=T_{\mathrm{e}}-T_{\mathrm{L}}-B_{\mathrm{m}} \omega_{\mathrm{r}}
$$

where $J$ is the moment of inertia; $T_{\mathrm{e}}$ and $T_{\mathrm{L}}$ are electromagnetic torque and load torque respectively; $B_{\mathrm{m}}$ is the linear viscous friction coefficient. Considering torque ripple, the torque is given as

$$
T_{\mathrm{e}}=T_{0}+\Delta T
$$

where, $T_{0}$ is the dc component of torque; $\Delta T$ is the sum of harmonic components of torque. Since the torque harmonics appear as the 1st, 2nd, 6th and multiples of 6th-order harmonic components, $\Delta T$ can be expressed as

$$
\begin{aligned}
\Delta T= & T_{1} \sin \left(p \omega_{\mathrm{r}} t+\theta_{1}\right)+T_{2} \sin \left(2 p \omega_{\mathrm{r}} t+\theta_{2}\right) \\
& +\sum_{i=1}^{\infty} T_{6 i} \sin \left(6 i p \omega_{\mathrm{r}} t+\theta_{6 i}\right)
\end{aligned}
$$

Conventional PMSM vector control system employs PI controller. Because of the limitation of the controller bandwidth, most harmonics are not effectively suppressed, as is analyzed in the next part.

\section{Conventional PMSM Vector Control}

In the conventional dual-closed-loop PMSM vector control system, PI controller is used for both speed and current control. The schematic of the control system is shown in Fig. 1.

As the time constant of inner loop is much smaller than that of the outer loop, assume sufficient current tracking accuracy, namely, $T_{\mathrm{e}}=K_{\mathrm{e}} i_{\mathrm{q}}=K_{\mathrm{e}} i_{\mathrm{q}}{ }^{*}$, and $K_{\mathrm{e}}=3 p \psi_{\mathrm{f}} / 2$ is the coefficient of motor torque. The inner loop can be treated as a forward link, represented by a constant, 1 . The reduced schematic of the control system is shown in Fig. 2.

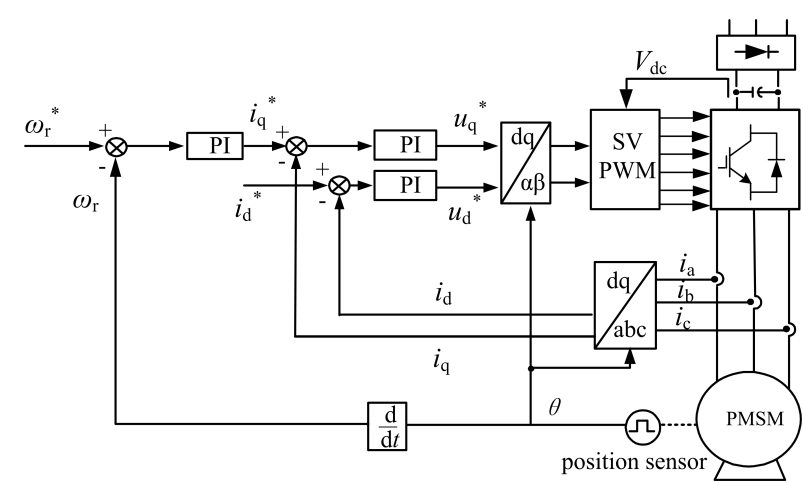

Fig. 1. Schematic of the vector control system of PMSM 


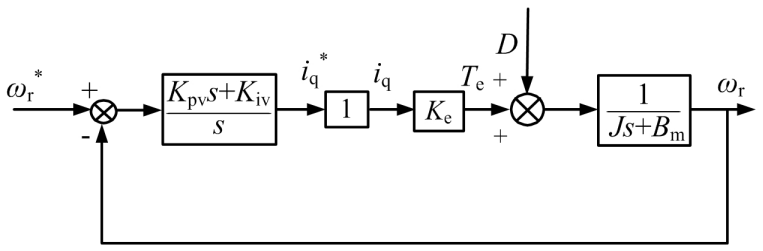

Fig. 2. Transfer function of vector control system

In the figure, $K_{\mathrm{pv}}$ and $K_{\mathrm{iv}}$ are respectively the proportional and integral coefficients of PI controller; $D$ is the equivalent disturbance, which consists of the periodic disturbances of different frequencies mentioned above, and load torque, etc.. Then the closed loop transfer function from disturbance $D(\mathrm{~s})$ to output $\omega_{\mathrm{r}}(\mathrm{s})$ can be shown as

$$
\varphi_{\mathrm{N}}(s)=\frac{\omega_{\mathrm{r}}(s)}{D(s)}=\frac{s}{J s^{2}+\left(B_{\mathrm{m}}+K_{\mathrm{pv}} K_{\mathrm{e}}\right) s+K_{\mathrm{iv}} K_{\mathrm{e}}}
$$

The amplitude-frequency characteristic can be obtained with $s=\mathrm{j} \omega$.

$$
\left|\varphi_{\mathrm{N}}(\mathrm{j} \omega)\right|=\frac{\omega}{\sqrt{\left(K_{\mathrm{iv}} K_{\mathrm{e}}-J \omega^{2}\right)^{2}+\omega^{2}\left(B_{\mathrm{m}}+K_{\mathrm{pv}} K_{\mathrm{e}}\right)^{2}}}
$$

Seen from the above equation, for the disturbance component of any frequency $\omega$ among $D(s)$, The larger the value of $K_{\mathrm{pv}}$ is, the smaller the amplitude of output response $\omega_{\mathrm{r}}(s)$ is, that is, the stronger the effect of disturbance suppression is. $K_{\mathrm{pv}}$ is the gain of PI controller, its value corresponds to the bandwidth of the system, which is limited by the hardware of the actual system. Too large bandwidth could cause low stability of the system, and thus $K_{\mathrm{pv}}$ is restricted to only a certain range of values, leading to inadequate suppression of the disturbance.

As is stated, the frequencies of the torque harmonics are integer multiples of the fundamental frequency of stator currents, $p \omega_{\mathrm{r}}$. If iterative learning control is adopted, the value of the controller gain can be made large enough only at these specific frequencies. Therefore, satisfactory suppression of the disturbances can be realized without affecting the stability of the system at the same time.

\section{PMSM Iterative Learning Control}

The main idea of iterative learning control(ILC) is that by using the previous iteration cycle control experience and system output error of the control system, the control signal of the next iteration cycle is generated based on iterative learning law, and the iteration is repeated again and again, before finally the desired output of the controlled object is obtained.

The Iterative learning law is

$$
u_{j+1}(t)=\alpha u_{j}(t)+\phi e_{j+1}(t)
$$

where, $j$ is the iteration number, $u_{j}(t)$ is the control signal, $e_{j}(t)$ is the output error of the controlled object; $\phi$ is the learning gain, $\alpha$ is the relaxation factor, aimed at weakening the accumulative effect of non-periodic disturbances. The necessary and sufficient condition for the convergence of the iterative error is $\alpha=1$ or $|\alpha|<1$ [22].

\subsection{ILC torque ripple suppression and controller parameter impact analysis}

Due to its control principle, when ILC is used to control the PMSM, in order to have a suppression effect on torque ripple, the period of iteration cycle should be consistent with that of the torque ripple, as is analyzed as follows.

When the motor is running at steady state, the time interval between the input and output of the ILC memory unit is equivalent to the period of iterative cycle, $\tau$, and it can be regarded as a delay link, $\mathrm{e}^{-\tau \mathrm{s}}$. Substituting ILC for the PI controller in the vector control system, we get the system shown in Fig. 3.

In the figure, the ILC link can be expressed as equivalent to $\phi /\left(1-\alpha \mathrm{e}^{-\tau s}\right)$. Thus the transfer function from the disturbance $D(s)$ to the output $\omega_{\mathrm{r}}(s)$ can be obtained as

$$
\varphi_{\mathrm{N}}(s)=\frac{\omega_{\mathrm{r}}(s)}{D(s)}=\frac{\left(1-\alpha \mathrm{e}^{-\tau s}\right)}{\left(J s+B_{\mathrm{m}}\right)\left(1-\alpha \mathrm{e}^{-\tau s}\right)+K_{\mathrm{e}} \phi}
$$

Known from the above analysis, each harmonic frequency of the torque ripple components is integer multiples of the stator current fundamental frequency, $p \omega_{\mathrm{r}}$. Thus the first-order harmonic cycle can be regarded as a common cycle of each harmonic. Take $\tau$ equal to the period of the first-order harmonic of torque ripple

$$
\tau=2 \pi / \omega_{\mathrm{e}}
$$

where $\omega_{\mathrm{e}}=p \omega_{\mathrm{r}}$ is the electrical angular velocity of the motor. As for (10), consider the input signal with frequency of $n \omega_{\mathrm{e}}$, where $n$ is a positive integer, thus $s=\mathrm{j} n \omega_{\mathrm{e}}$, then

$$
\mathrm{e}^{-\tau S}=\mathrm{e}^{-\mathrm{j} n \omega \tau}=\mathrm{e}^{-\mathrm{j} 2 n \pi}=1
$$

Thus (10) can be simplified as

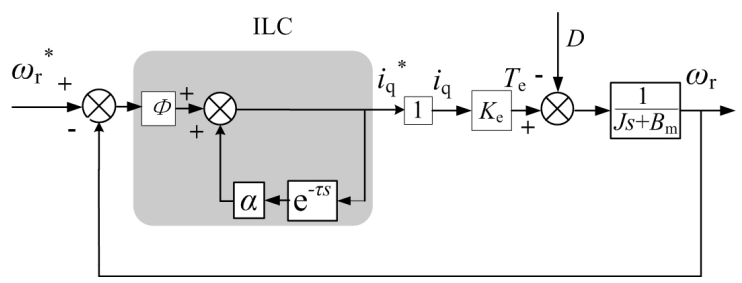

Fig. 3. Transfer function of iterative learning control system 


$$
\varphi_{\mathrm{N}}\left(\mathrm{j} n \omega_{\mathrm{e}}\right)=\frac{1-\alpha}{\left(\mathrm{j} n \omega_{\mathrm{e}} J+B_{\mathrm{m}}\right)(1-\alpha)+K_{\mathrm{e}} \phi}
$$

When $\alpha=1$, (13) equals 0 , meaning that the amplitude of the system output response to the disturbance component with frequency of $n \omega_{\mathrm{e}}$ is 0 , and that the $n$-th order harmonic of the torque ripple is completely suppressed. Thus in order to effectively suppress the torque ripple, the value of $\alpha$ should be adequately close to 1 . As stated in [22], the absolute value of $\alpha$ is less than 1 for the convergence of the algorithm, thus a larger value of $\alpha$ within the range of $(0,1]$ is usually better for suppression of periodic disturbance.

However, ILC is not able to suppress non-periodic disturbance. It can be seen from (9) that with larger value of $\alpha$ in the range of $(0,1]$, the control signal of the current iteration cycle $u_{j}(t)$ will have greater influence on that of the next iteration cycle $u_{j+1}(t)$. This will lead to more significant accumulative effect of non-periodic disturbance contained in the signal. In severe cases, it may even cause the divergence of the algorithm. In addition, with larger value of $\alpha$, dynamic response of the system will be worsened. As is seen from (9), if the value of $\alpha$ is close to 1 , the control signal of each iteration is almost the same as the previous iteration in the steady state. The system now presents approximately periodicity, and the control signal of an iteration cycle is almost completely retained and delivered to the next iteration cycle. Once the system enters the dynamic process caused by, for example, speed step or load torque step, the system fails to present the periodicity any longer. If at this time the control signal from the previous iteration cycle is still retained almost completely, it will inevitably lead to greater fluctuation or longer regulating time of the dynamic process. Therefore, in consideration of dynamic process and convergence of ILC, the value of $\alpha$ can not be too close to 1 , and a fairly small value within $(0,1)$ is more appropriate.

Thus, the value of $\alpha$ has a great impact on both steady state and dynamic performance of the system. In order to adequately suppress the torque ripple under steady state, while prevent evident deterioration of dynamic performance, a parameter optimization based iterative learning control is proposed, online adjusting the value of the parameter $\alpha$ according to the principle of minimum error, selecting an appropriate value of $\alpha$ in steady-state and dynamic process respectively.

As can be seen from (10), the learning gain $\phi$ of ILC also has an impact on disturbance suppression, and larger value of $\phi$ will have better effect on reducing the torque ripple. But this effect is not aimed at disturbances at specific frequencies. $\phi$ is a proportional coefficient of the error, its role is similar to that of the proportional coefficient $K_{\mathrm{pv}}$ of PI controller, and its value is also restricted by the hardware system. The tuning of $\phi$ can be implemented in two steps. Firstly, restrict the range of $\phi$ according to the requirement of stability margin. Obtain the open-loop transfer function of the speed loop in consideration of speed measuring delay, calculate the openloop frequency characteristic, and referring to the phasefrequency characteristic, the cut-off frequency should be selected much lower than that of the measuring delay to guarantee the phase margin. Secondly, select the bandwidth of the speed loop, and $\phi$ is determined accordingly, which must meet the requirement of stability margin in the first step.

\subsection{Parameter optimization based ILC}

The adjustment of the parameter $\alpha$ in iterative learning controller is intended to construct a more reasonable control signal of the next iteration cycle so that the controlled object output error is as small as possible. Due to the periodicity of disturbances or input signal, the system input and output error should be considered within a complete iteration cycle. The control unit in practical application is a digital signal processor, of which the control step size (or sampling period ) is represented by $T_{\mathrm{s}}$, so the number of control steps in an iteration cycle is $N=\tau / T_{\mathrm{s}}$. To adjust the parameter $\alpha$, the functional relationship between the system output error and $\alpha$ of each step within an iteration cycle should be obtained, therefore the controlled object is discretized.

At no load, discretizing (4), while considering periodic disturbances, we get

$$
\omega_{\mathrm{r}}(k+1)=\left(1-\frac{T_{\mathrm{s}} B_{\mathrm{m}}}{J}\right) \omega_{\mathrm{r}}(k)+\frac{T_{\mathrm{s}} K_{\mathrm{e}}}{J} i_{\mathrm{q}}(k)+w(k)
$$

Take the system state variable as $x(k)=\omega_{\mathrm{r}}(k)$, system input as $u(k)=i_{\mathrm{q}}(k)$, and output as $y(k)=\omega_{\mathrm{r}}(k), \quad A=1$ $\left(T_{\mathrm{s}} B_{\mathrm{m}} / J\right), B=T_{\mathrm{s}} K_{\mathrm{e}} / J$, we get

$$
\left\{\begin{array}{l}
x(k)=A x(k-1)+B u(k-1)+w(k-1) ; \\
y(k)=x(k) ; \quad k=1,2,3, \ldots, N
\end{array}\right.
$$

where $w(k)$ is periodic disturbance. Through iterations, we get

$$
\begin{aligned}
y(k) & =x(k) \\
& =A x(k-1)+B u(k-1)+w(k-1) \\
& =A[A x(k-2)+B u(k-2)+w(k-2)] \\
& +B u(k-1)+w(k-1) \\
& \vdots \\
& =A^{k} x(0)+\sum_{i=0}^{k-1} A^{k-i-1} B u(i)+\sum_{i=0}^{k-1} A^{k-i-1} w(i)
\end{aligned}
$$

Usually it is assumed that the initial state $x(0)=0$. When $k$ is respectively taken as $1,2, \ldots \ldots, N$, a set of $N$ equations 
is obtained, which is the expression of the system output of each step, and can be also expressed in matrix form as

$$
\boldsymbol{y}=\boldsymbol{P u}+\boldsymbol{Q w}
$$

where, $\boldsymbol{u}=[u(0) u(1) \ldots u(N-1)]^{\mathrm{T}}, \boldsymbol{y}=[y(1) y(2) \ldots y(N)]^{\mathrm{T}}$,

$$
\begin{aligned}
& \boldsymbol{P}=\left[\begin{array}{ccccc}
\boldsymbol{w}=[w(0) & w(1) & \ldots & w(N-1)]^{\mathrm{T}}, \\
B & 0 & 0 & \cdots & 0 \\
A B & B & 0 & \cdots & 0 \\
A^{2} B & A B & B & \ddots & 0 \\
\vdots & \vdots & \vdots & \ddots & \vdots \\
A^{N-1} B & A^{N-2} B & A^{N-3} B & \cdots & B
\end{array}\right], \\
& \boldsymbol{Q}=\left[\begin{array}{ccccc}
1 & 0 & 0 & \cdots & 0 \\
A & 1 & 0 & \cdots & 0 \\
A^{2} & A & 1 & \ddots & 0 \\
\vdots & \vdots & \vdots & \ddots & \vdots \\
A^{N-1} & A^{N-2} & A^{N-3} & \cdots & 1
\end{array}\right] .
\end{aligned}
$$

(17) is the expression of system output within an iteration cycle. For the $j$-th and $(j+1)$-th iteration, there is

$$
\left\{\begin{array}{l}
\boldsymbol{y}_{j}=\boldsymbol{P} \boldsymbol{u}_{j}+\boldsymbol{Q} \boldsymbol{w}_{j} \\
\boldsymbol{y}_{j+1}=\boldsymbol{P} \boldsymbol{u}_{j+1}+\boldsymbol{Q} \boldsymbol{w}_{j+1}
\end{array}\right.
$$

The diagram of relationship between iteration cycle $\tau$ and control step $T_{\mathrm{s}}$ is shown in Fig. 4.

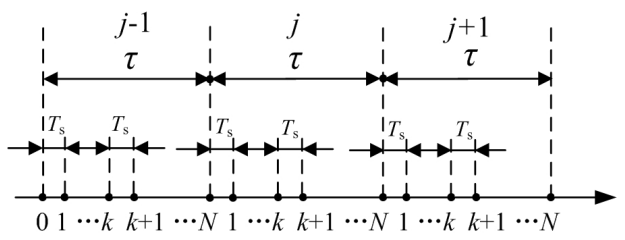

Fig. 4. Diagram of iteration cycle

As the period of iteration cycle is set equal to that of the disturbance, thus the disturbance appears the same over different iteration cycles, namely $\boldsymbol{w}_{j}=\boldsymbol{w}_{j+1}=\boldsymbol{w}$. System output error is

$$
\left\{\begin{array}{l}
\boldsymbol{e}_{j}=\boldsymbol{y}^{*}-\boldsymbol{y}_{j}=\left(\boldsymbol{y}^{*}-\boldsymbol{Q w}\right)-\boldsymbol{P} u_{j} \\
\boldsymbol{e}_{j+1}=\boldsymbol{y}^{*}-\boldsymbol{y}_{j+1}=\left(\boldsymbol{y}^{*}-\boldsymbol{Q w}\right)-\boldsymbol{P} u_{j+1}
\end{array}\right.
$$

where, $\boldsymbol{y}^{*}=\left[y^{*}(1) y^{*}(2) \ldots y^{*}(N)\right]^{\mathrm{T}}$ is the reference of system output. As $\left(\boldsymbol{y}^{*}-\boldsymbol{Q w}\right)$ is constant with respect to the iteration number $j$, replace $\left(\boldsymbol{y}^{*}-\boldsymbol{Q} \boldsymbol{w}\right)$ in (19) with a new symbol $\boldsymbol{y}_{\mathrm{d}}{ }^{*}$.The iterative learning law is

$$
\boldsymbol{u}_{j+1}=\alpha_{j+1} \boldsymbol{u}_{j}+\boldsymbol{L} \boldsymbol{e}_{j+1}
$$

where $\boldsymbol{L}=\operatorname{diag}(\phi)$ is the learning gain matrix.

From $(18) \sim(20)$, we get

$$
(\boldsymbol{I}+\boldsymbol{P} \boldsymbol{L}) \boldsymbol{e}_{j+1}=\left(1-\alpha_{j+1}\right) \boldsymbol{y}_{\mathrm{d}}{ }^{*}+\alpha_{j+1} \boldsymbol{e}_{j}
$$

where $\boldsymbol{I}$ is the identity matrix of the order of $N$. (21) is the needed functional relationship between the system output error and $\alpha_{j+1}$. In order to select the appropriate value of $\alpha_{j+1}$, The optimization problem is structured as

$$
\left\{\begin{array}{l}
\min _{0<\alpha_{j+1}<1} f\left(\alpha_{j+1}\right) \\
f\left(\alpha_{j+1}\right)=\left\|\left(1-\alpha_{j+1}\right) \boldsymbol{y}_{\mathrm{d}}{ }^{*}+\alpha_{j+1} \boldsymbol{e}_{j}\right\|_{\infty}+K_{\alpha}\left|\alpha_{j+1}\right|^{2}
\end{array}\right.
$$

where $\|\cdot\|_{\infty}$ is the infinite norm. As the infinite norm of matrices is consistent with that of vectors, from (21) we have

$$
\left\|\boldsymbol{e}_{j+1}\right\|_{\infty} \leq\left\|(\boldsymbol{I}+\boldsymbol{P} \boldsymbol{L})^{-1}\right\|_{\infty}\left\|\left(1-\alpha_{j+1}\right) \boldsymbol{y}_{\mathrm{d}}^{*}+\alpha_{j+1} \boldsymbol{e}_{j}\right\|_{\infty}
$$

As $(\boldsymbol{I}+\boldsymbol{P L})$ is a constant matrix, it can be seen that on the right side of (22), the first term $\left\|(1-\alpha) \boldsymbol{y}_{\mathrm{d}}{ }^{*}+\alpha \boldsymbol{e}_{j}\right\|_{\infty}$ is meant to minimize $\left\|\boldsymbol{e}_{j+1}\right\|_{\infty}$, the error estimate in the next iteration cycle; while the second term is to prevent $\alpha_{j+1}$ from being too large a value, so as to reduce the accumulation of the non-periodic disturbance. $K_{\alpha}$ is used to adjust the weights of these two terms, of which the weight ratio is $1: K_{\alpha}$.

To simplify the calculation, consider only the situation where the reference output value is positive, and ignore the overshoot of the system response, thus (22) can be written as

$$
f\left(\alpha_{j+1}\right)=\left(1-\alpha_{j+1}\right)\left\|y_{\mathrm{d}}{ }^{*}\right\|_{\infty}+\alpha_{j+1}\left\|\boldsymbol{e}_{j}\right\|_{\infty}+K_{\alpha}\left|\alpha_{j+1}\right|^{2} .
$$

To solve for the optimal value of $\alpha_{j+1}$, according to the principle of extreme value method, calculate the derivation of (24)

$$
\mathrm{d} f\left(\alpha_{j+1}\right) / \mathrm{d} \alpha_{j+1}=-\left\|\boldsymbol{y}_{\mathrm{d}}^{*}\right\|_{\infty}+\left\|\boldsymbol{e}_{j}\right\|_{\infty}+2 K_{\alpha} \alpha_{j+1}
$$

Set the equation zero, and the solution is

$$
\alpha_{j+1}=\left(\left\|y_{\mathrm{d}}^{*}\right\|_{\infty}-\left\|\boldsymbol{e}_{j}\right\|_{\infty}\right) / 2 K_{\alpha}
$$

The weighting factor $K_{\alpha}$ should be chosen properly in accordance with the system response. During a dynamic process, the system output error $\left\|\boldsymbol{e}_{j}\right\|_{\infty}$ is large, thus it is necessary to increase $K_{\alpha}$, the weight of the second term, so as to reduce the accumulation of non-periodic disturbance. In steady state, $\left\|\boldsymbol{e}_{j}\right\|_{\infty}$ is small, and the periodic disturbance 


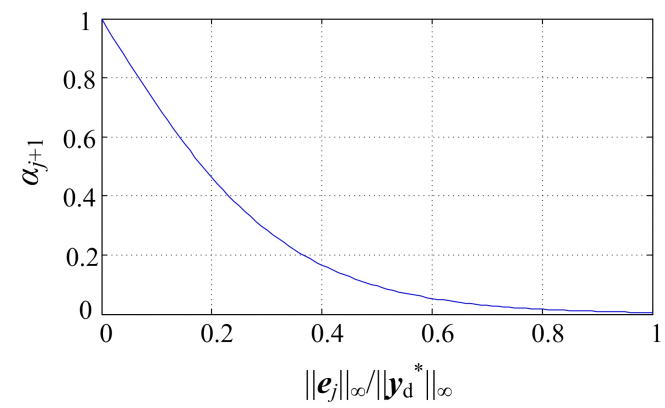

Fig. 5. Functional relationship between $\alpha_{j+1}$ and $\left\|e_{j}\right\|_{\infty}$

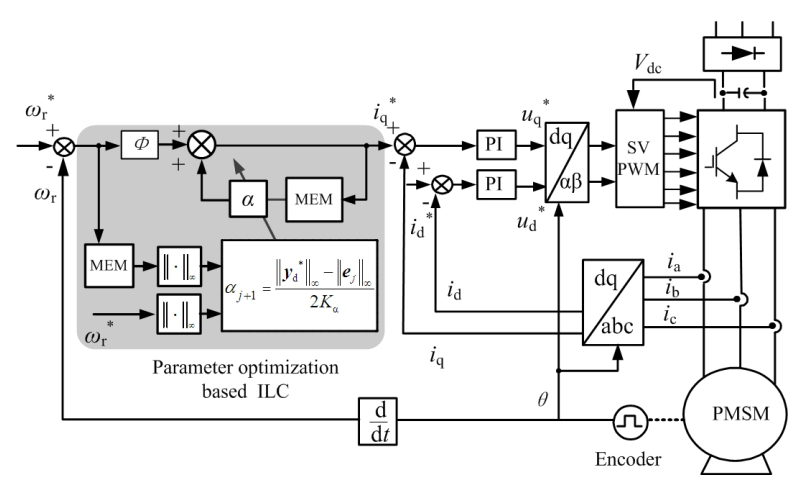

Fig. 6. Diagram of PMSM control system using parameter optimization based ILC

turns into the principal component, so a smaller value of $K_{\alpha}$ is selected to increase the weight of the first term of (22). Therefore, according to (26) and the variation of $K_{\alpha}$, the relationship between $\alpha_{j+1}$ and $\left\|\boldsymbol{e}_{j}\right\|_{\infty}$ is approximated by the diagram in Fig. 5.

The diagram of PMSM control system using parameter optimization based ILC is shown in Fig. 6.

\subsection{Stability analysis}

According to (9) and Fig. 3. the q-axis current in $\mathrm{z}$ domain can be expressed as

$$
i_{\mathrm{q}, j+1}^{*}(z)=\alpha i_{\mathrm{q}, j}^{*}(z)+\phi e_{j+1}(z)
$$

The iteration process in ILC is treated as time delay process

$$
z^{-N} i_{\mathrm{q}, j+1}^{*}(z)=i_{\mathrm{q}, j}^{*}(z)
$$

where $N=\tau / T_{\mathrm{s}}$ is the number of control steps in an iteration cycle. Thus the transfer function of ILC in z-domain is

$$
G(z)=\frac{i_{\mathrm{q}, j+1}^{*}(z)}{e_{j+1}(z)}=\frac{\phi z^{N}}{z^{N}-\alpha}
$$

From (29), all the poles of the transfer function $G(z)$ satisfy $|z|^{N}=|\alpha|$. As the value of $\alpha$ is within the range of $(0,1)$, thus $|\alpha|<1$, and therefore $|z|<1$, namely all the poles of $G(z)$ are located inside the unit circle in the $\mathrm{z}$ plane, indicating that the controller is stable.

\section{Experiment Results and Analysis}

To verify the feasibility and effectiveness of the proposed algorithm, experiments are carried out on a $5.2 \mathrm{~kW}$ low speed high torque PMSM. The parameters of the controlled PMSM are given in Table 1. The Power Device of the experimental system is PM75DSA120, and the switching frequency is $5 \mathrm{kHz}$. The MCU is TMS320F28335, and the sampling period is $100 \mu \mathrm{s}$. The controlled motor is coaxially connected to generator load. The load machine is also a PMSM, its stator is connected to three phase resistance through an ac contactor. The experimental setup is shown in Fig. 7.

Table 1. Parameter of the controlled PMSM

\begin{tabular}{cc||cc}
\hline Parameter & value & Parameter & value \\
\hline Rate voltage $U_{\mathrm{N}}(\mathrm{V})$ & 380 & Rate speed $n_{\mathrm{N}}(\mathrm{r} / \mathrm{min})$ & 50 \\
Rate power $P_{\mathrm{N}}(\mathrm{kW})$ & 5.2 & Stator resistance $R_{\mathrm{s}}(\Omega)$ & 1.89 \\
Rate torque $T_{\mathrm{N}}(\mathrm{N} \cdot \mathrm{m})$ & 1000 & Stator inductance $L_{\mathrm{s}}(\mathrm{mH})$ & 45 \\
Inertia $J(\mathrm{~kg} \cdot \mathrm{m} 2)$ & 3.0 & PM flux $\psi_{\mathrm{f}}(\mathrm{Wb})$ & 1.63 \\
Pole pairs $p$ & 24 & & \\
\hline
\end{tabular}

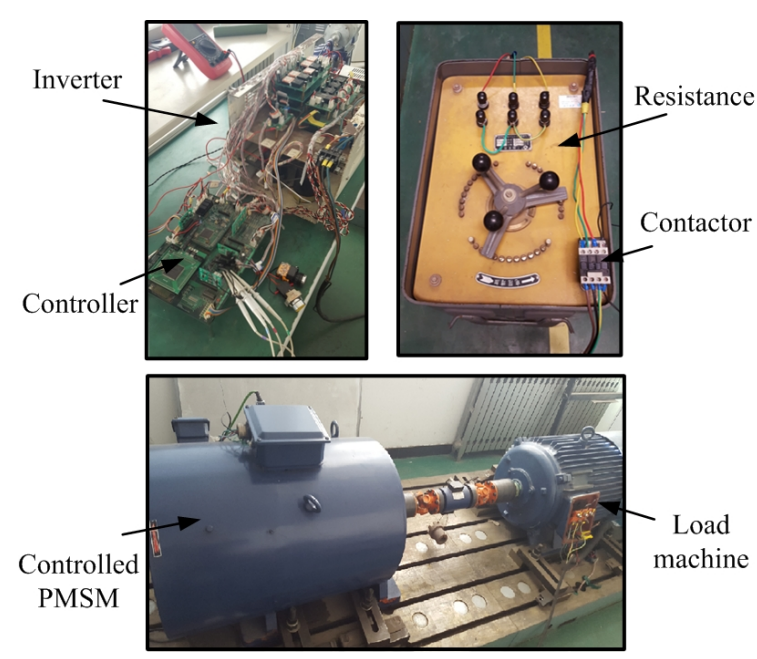

Fig. 7. Experimental setup

\subsection{Steady state experiment}

To verify the torque ripple suppression effect of ILC, steady state experiments are carried out firstly. Fig. 8(a) shows speed, torque and current curves when using the PI controller, and Fig. 8(b) shows the curves when using the iterative learning controller. The speed of the motor is $20 \mathrm{r} / \mathrm{min}$, the load torque is $140 \mathrm{~N} . \mathrm{m}$. The parameters of PI controller in speed loop are $K_{\mathrm{pv}}=2.54, K_{\mathrm{iv}}=0.02$. The parameters of PI controller in current loop are $K_{\mathrm{pi}}=48.99$, 

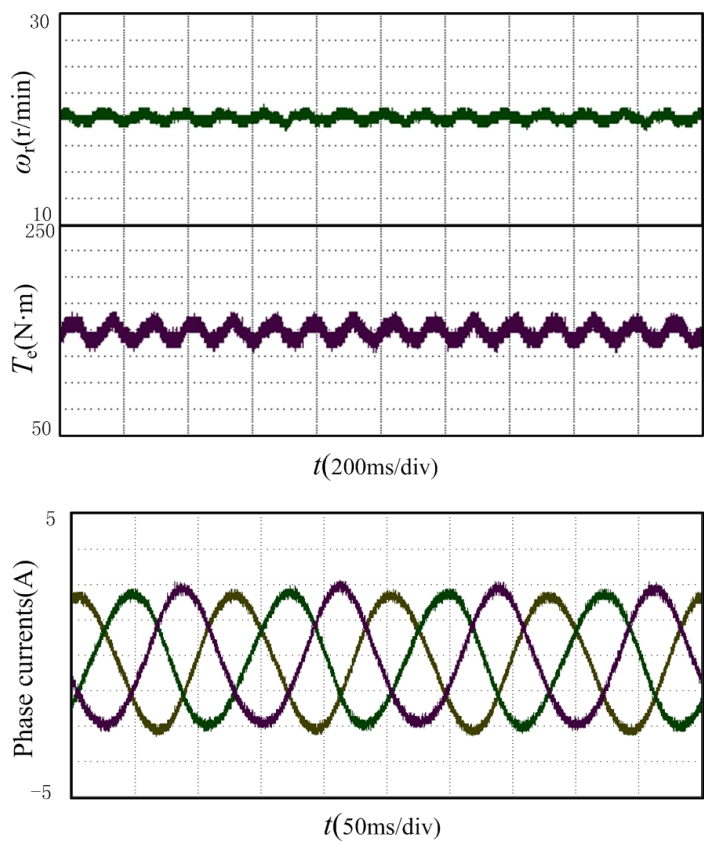

(a)
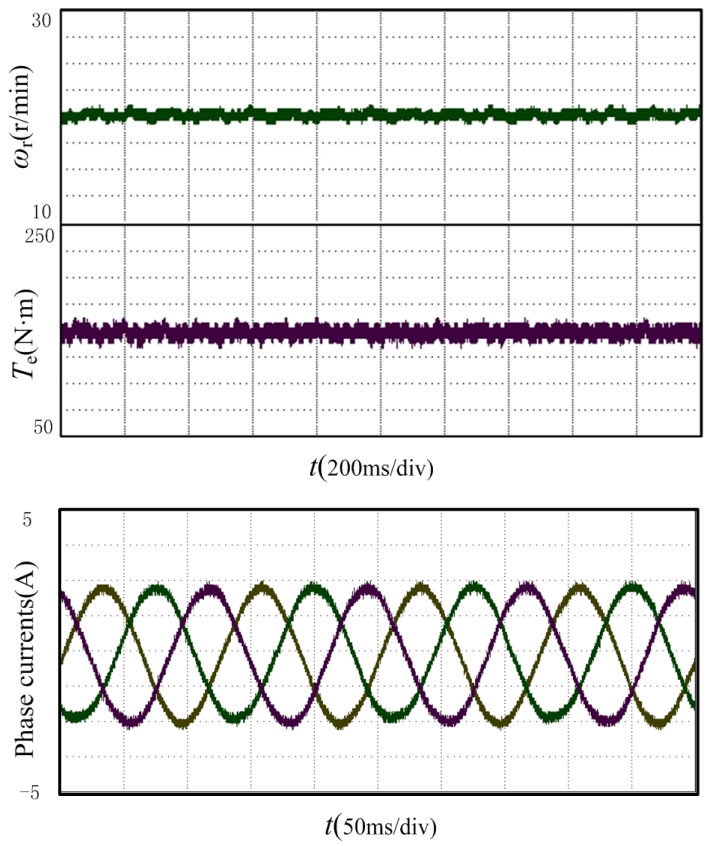

(b)

Fig. 8. Steady state response: (a) PI controller; (b) iterative learning controller

$K_{\mathrm{ii}}=1200$. The parameters of iterative learning controller are $\alpha=0.9, \phi=2.54$.

The torque ripple factor and speed ripple factor are defined as

$$
\left\{\begin{array}{l}
T R F=T_{\mathrm{pp}} / T_{\mathrm{av}} \\
S R F=\omega_{\mathrm{pp}} / \omega_{\mathrm{av}}
\end{array}\right.
$$

where $T_{\mathrm{pp}}$ and $\omega_{\mathrm{pp}}$ are the peak-peak value of torque and speed respectively; $T_{\mathrm{av}}, \omega_{\mathrm{av}}$ are the mean value of torque and speed respectively. Calculation on the basis of Fig. 8(a) and $8(\mathrm{~b})$ shows that, the torque ripple factor is reduced to $4.74 \%$ using the ILC, compared with $9.65 \%$ using the PI controller; the speed ripple factor is reduced to $1.95 \%$ using ILC, compared with $4.65 \%$ using PI controller. It can be seen that ILC controller has better effect on suppressing the torque and speed ripples than the PI controller does.

To explore the effect that the value of $\alpha$ has on speed and torque ripple suppression, experiments are carried out with different value of $\alpha$ in steady state of $20 \mathrm{r} / \mathrm{min}$ and $140 \mathrm{~N} . \mathrm{m}$ load torque. The torque ripple factor and speed ripple factor with different values of $\alpha$ are shown in Fig. 9. It shows that effect of ripple suppression is better with larger value of $\alpha$.

\subsection{Dynamic experiment}

To verify the improvement of dynamic performance, dynamic experiments are carried out using ILC with a fixed value of $\alpha$, ILC with adjustable $\alpha$ (namely parameter optimization based ILC) and PI controller respecitively.

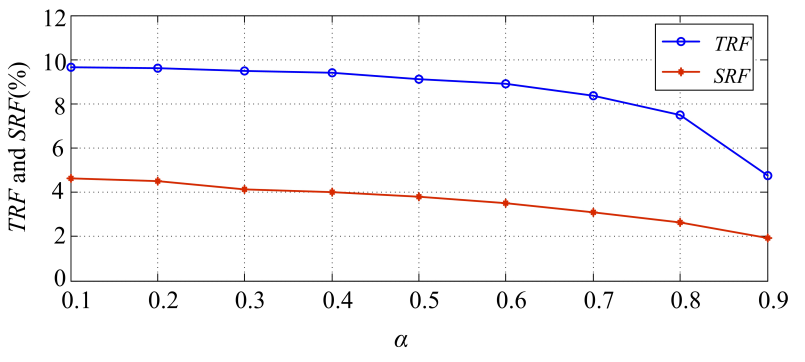

Fig. 9. $T R F$ and $S R F$ under different $\alpha$

The dynamic response curves using ILC with a fixed value of $\alpha=0.9$ are shown in Fig. 10. The response curves at load step from 0 to 140 N.m are shown in Fig. 10(a), including speed, integral square error (ISE) of speed, electromagnetic torque and load torque. In each iteration cycle, the square of speed error is cumulated at every control step, until the last control step of the iteration cycle, yielding ISE of that iteration cycle. Later the calculation enters the next iteration cycle and begins calculating ISE of the next cycle. The ISE in each iteration cycle (ISE ISE $_{j+1}$, $\left.\mathrm{ISE}_{j+2}\right)$ with respect to the iteration number $(j, j+1, j+2)$ after load step are labeled in the speed ISE curve in Fig. 10(a). The response curves of the speed step from 10r/min to $20 \mathrm{r} / \mathrm{min}$ at no load are shown in Fig. 10(b).

As can be seen from the figure, both speed and torque shows great fluctuation after the step, and then gradually converge to the steady state value, which is in accordance with the analysis in Part 4.1. During the load step dynamics, the regulating time for speed and torque, $t_{\mathrm{s}, \omega}$ and $t_{\mathrm{s}, T}$, are $0.83 \mathrm{~s}$ and $0.87 \mathrm{~s}$ respectively; while during the speed step dynamics, they are $1.03 \mathrm{~s}$ and $1 \mathrm{~s}$, which are as long as 8 


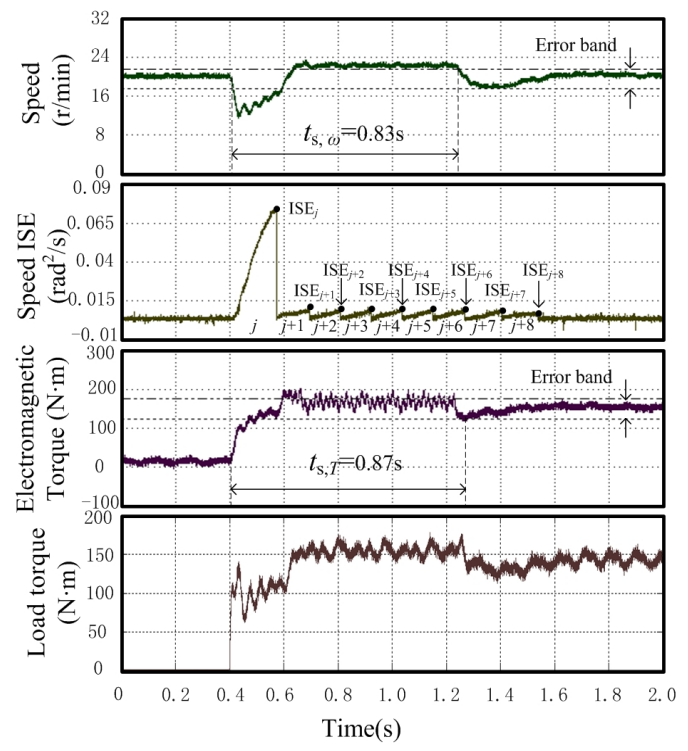

(a)

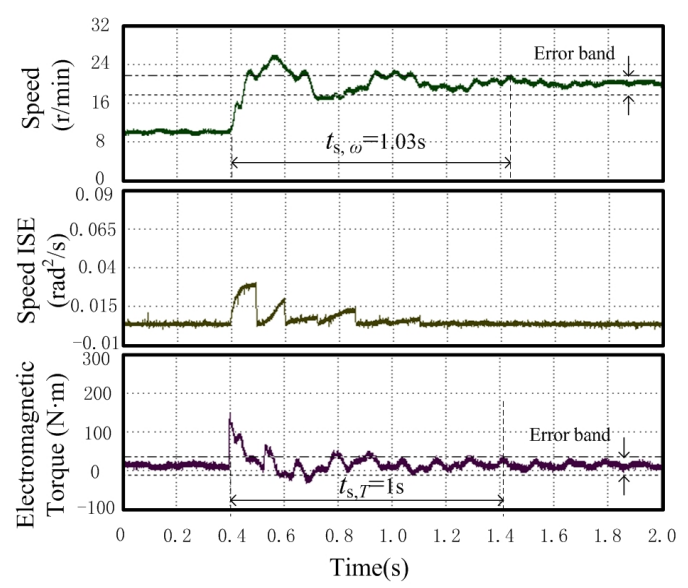

(b)

Fig. 10. Dynamic response curve using ILC with $\alpha=0.9$ (a) at load step from 0 to 140 N.m (b) at speed step from 10 to $20 \mathrm{r} / \mathrm{min}$

iteration cycles.

Fig. 11 shows the number of iteration cycles needed by regulating time before convergence (error less than 5\%) during speed stepping dynamic process at light load, under different values of $\alpha$. The figure shows that the larger the value of $\alpha$ is, the longer regulating time is needed.

Fig. 12 shows the dynamic response using parameter optimization based ILC during the load and speed step dynamic process. The curve of $\alpha$ is included in the figure. As $\alpha$ is adjustable, its value is different during the dynamic process. In contrast, $\phi$ is not adjustable, its value remains constant. The experimental condition is the same as that of Fig. 10. Fig 12(a) shows the response curves during the load step dynamics, and it can be seen that $t_{\mathrm{s}, \omega}$ and $t_{\mathrm{s}, T}$ are $0.39 \mathrm{~s}$ and $0.3 \mathrm{~s}$, respectively. Fig $12(\mathrm{~b})$ shows the response curves during the speed step dynamics, and it can be seen that $t_{\mathrm{s}, \omega}$ and $t_{\mathrm{s}, T}$ are both $0.2 \mathrm{~s}$. Compared with

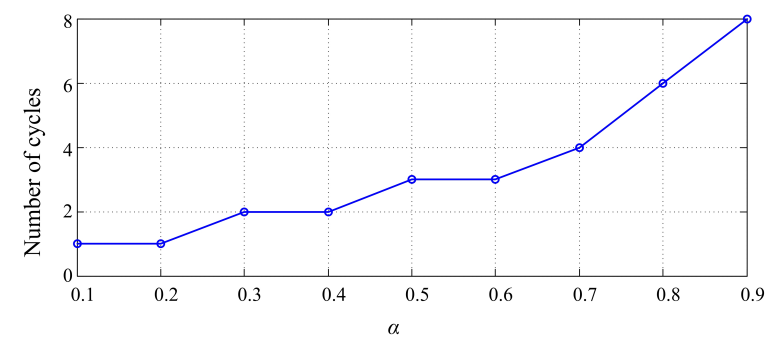

Fig. 11. Regulating time under different $\alpha$

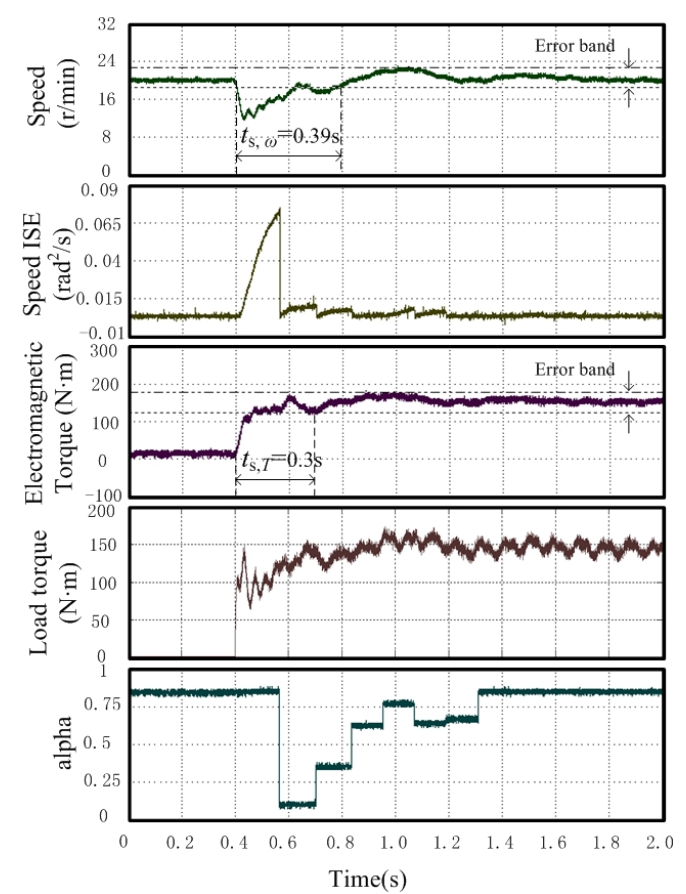

(a)

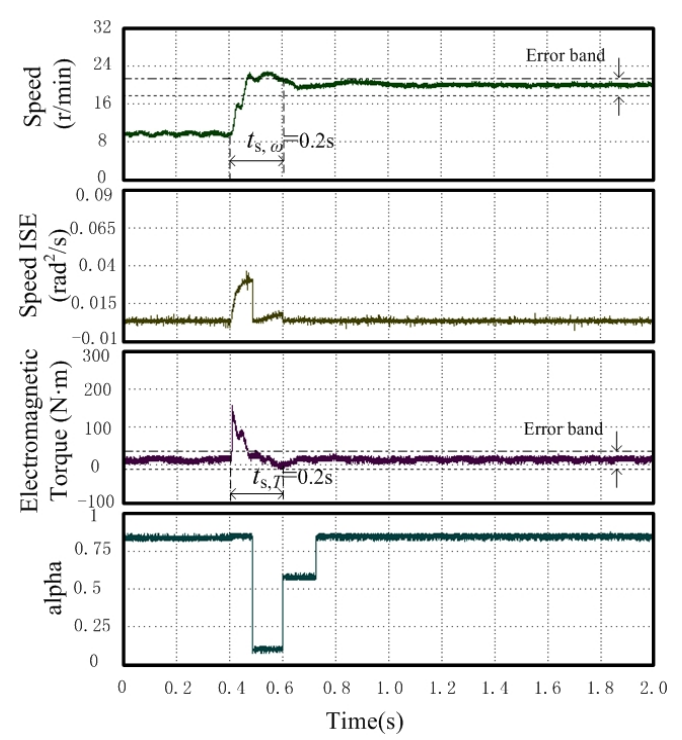

(b)

Fig. 12. Dynamic response curve using ILC with adjustable $\alpha$ : (a) at load step from 0 to 140 N.m; (b) at speed step from 10 to $20 \mathrm{r} / \mathrm{min}$ 


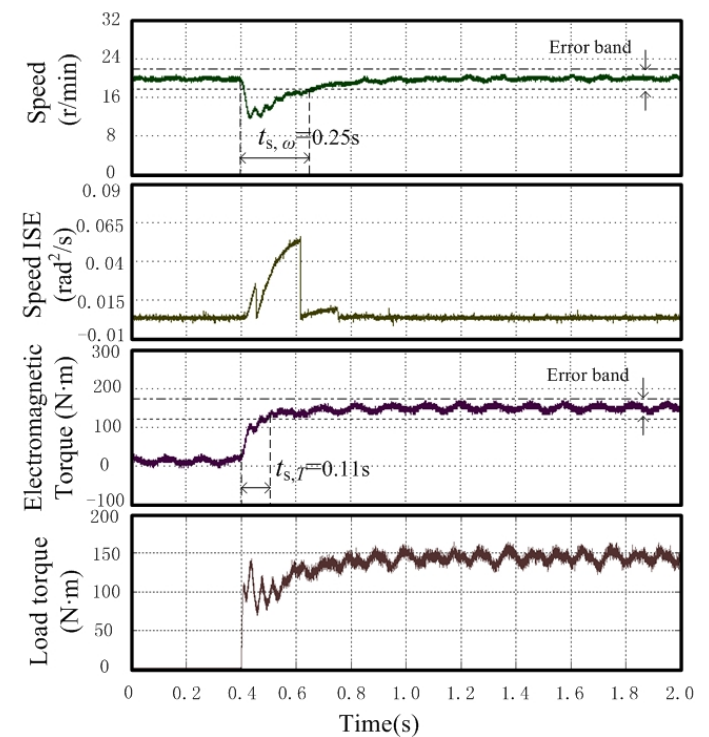

(a)

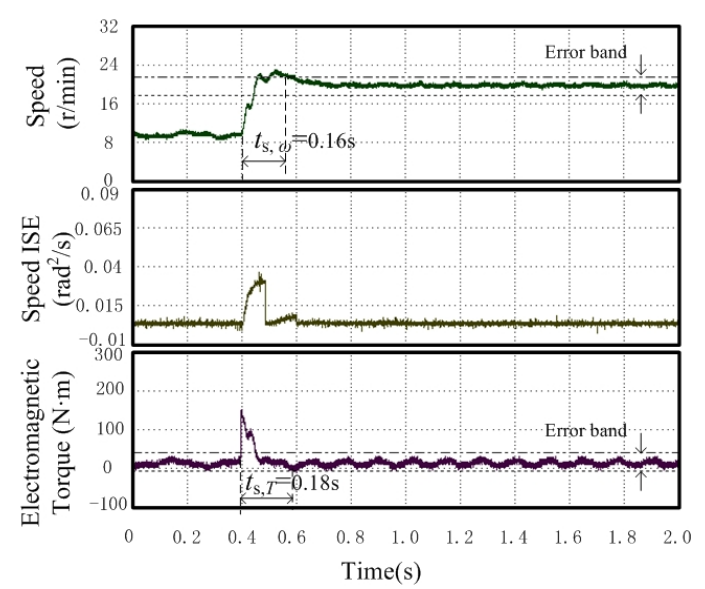

(b)

Fig. 13. Dynamic response curve using PI controller: (a) at load step from 0 to 140 N.m; (b) at speed step from 10 to $20 \mathrm{r} / \mathrm{min}$

Fig. 10, Fig. 12 shows much shorter regulating time, and that the dynamic response is significantly improved with adjustable $\alpha$.

Fig. 13 shows the dynamic response using PI controller, during the load and speed step dynamic process. The experimental condition is also the same as that of Fig. 10. and Fig. 12. Fig 13(a) shows the response curves during the load step dynamics, where $t_{\mathrm{s}, \omega}$ and $t_{\mathrm{s}, T}$ are $0.25 \mathrm{~s}$ and $0.11 \mathrm{~s}$, respectively. Fig 13(b) shows the response curves during the speed step dynamics, where $t_{\mathrm{s}, \omega}$ and $t_{\mathrm{s}, T}$ are $0.16 \mathrm{~s}$ and $0.18 \mathrm{~s}$ respectively. The results show that the regulating time of Fig. 12 is much closer to that of Fig. 13 than that of Fig. 10 is. Therefore, compared with PI controller, parameter optimization based ILC achieves significant reduction of torque and speed ripple on the one hand, and keeps fairly good dynamic performance on the other hand.

To verify the effectiveness of the proposed algorithm at

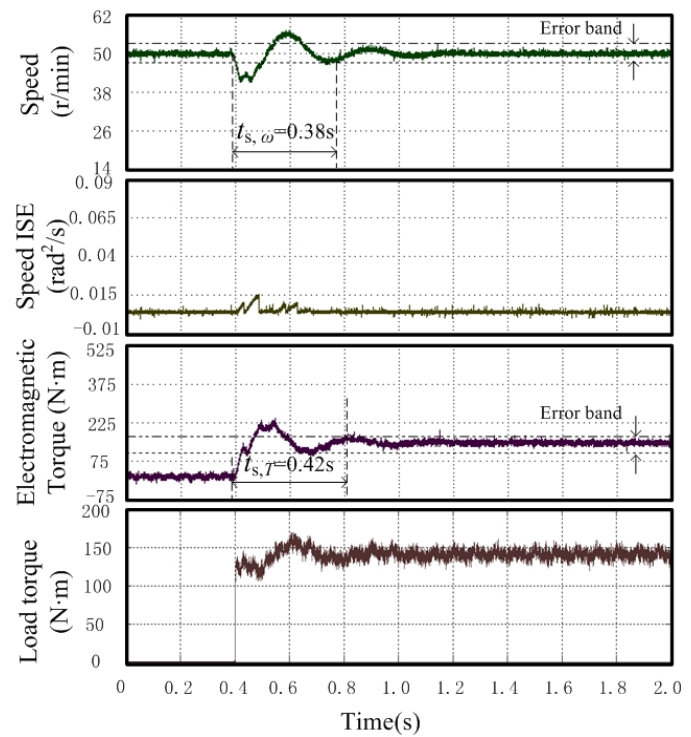

(a)

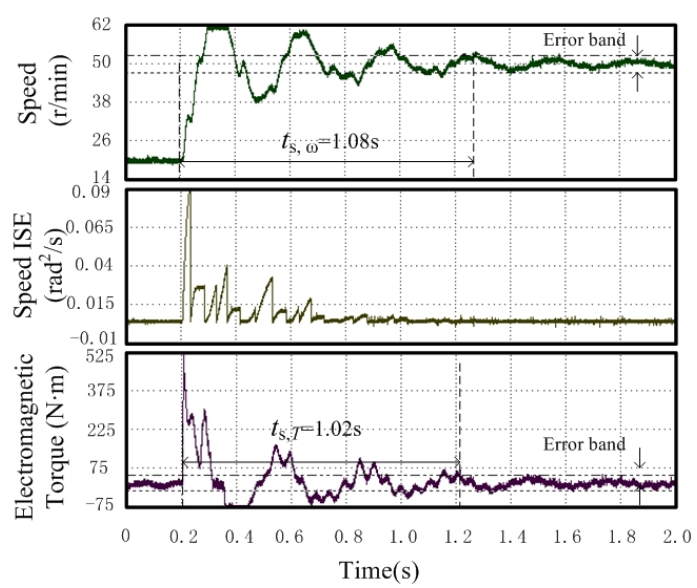

(b)

Fig. 14. Dynamic response curve using ILC with $\alpha=0.9$ : (a) at load step from 0 to 140 N.m; (b) at speed step from 20 to $50 \mathrm{r} / \mathrm{min}$

different operating condition, experiments are carried out at the rated speed 50r/min. Fig. 14(a) and 14(b) show the dynamic response curves respectively at load step from 0 to $140 \mathrm{~N} . \mathrm{m}$ and at speed step from $20 \mathrm{r} / \mathrm{min}$ to $50 \mathrm{r} / \mathrm{min}$ using ILC with a fixed value of $\alpha=0.9$; Fig. 15(a) and 15(b) show the curves using parameter optimization based ILC; Fig. 16(a) and 16(b) show the curves using PI controller. As can be seen from the figures, the regulating time when using parameter optimization based ILC is much shorter than that when using ILC with a fixed value of $\alpha$, and is much closer to that when using PI controller.

\section{Conclusion}

In regard to the problem of the periodic torque ripple of PMSM, this paper analyzed the effect of ILC controller on 


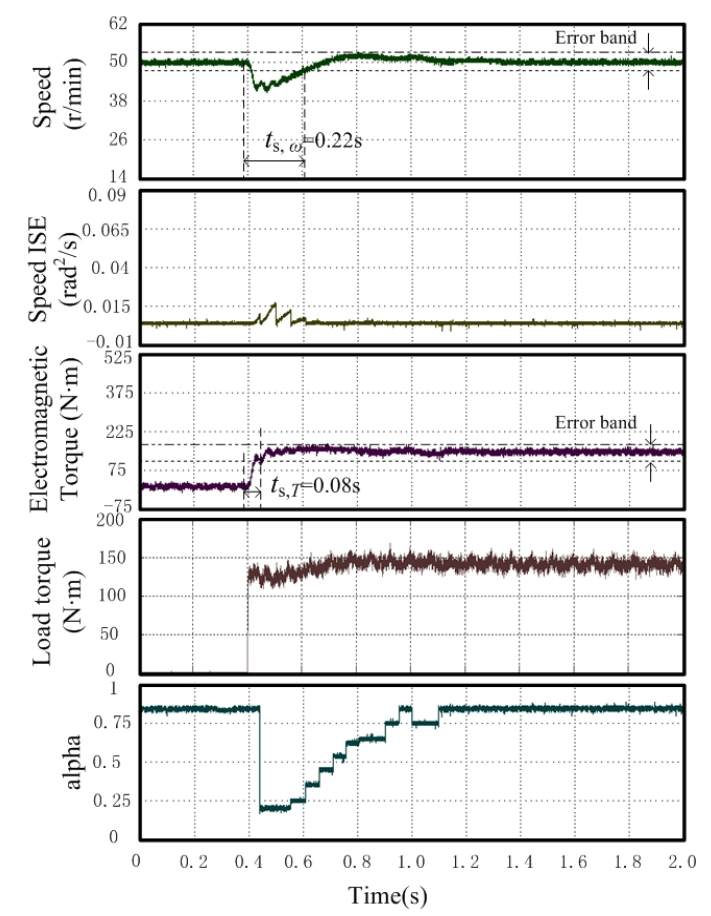

(a)

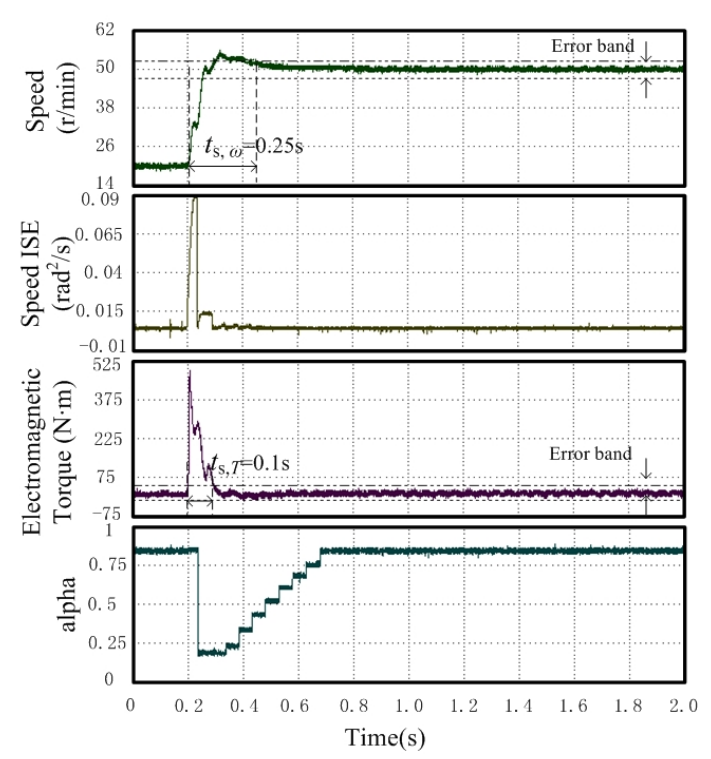

(b)

Fig. 15. Dynamic response curve using ILC with adjustable $\alpha$ : (a) at load step from 0 to $140 \mathrm{~N} . \mathrm{m}$; (b) at speed step from 20 to $50 \mathrm{r} / \mathrm{min}$

torque ripple suppression compared with PI controller. The parameter $\alpha$ of ILC is revealed to have impact on both suppression effect during steady state and the regulating time during dynamic process. A parameter optimization based iterative learning control is proposed, online adjusting the parameter $\alpha$ based on the optimal solution. The purpose of reducing the torque ripple is achieved, while the convergence rate and the dynamic performance of the system is improved compared with ILC with fixed

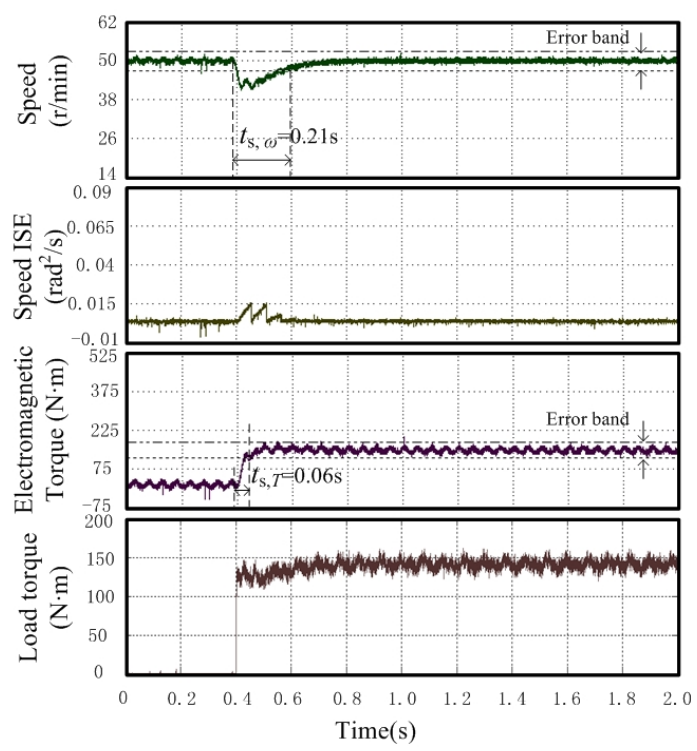

(a)

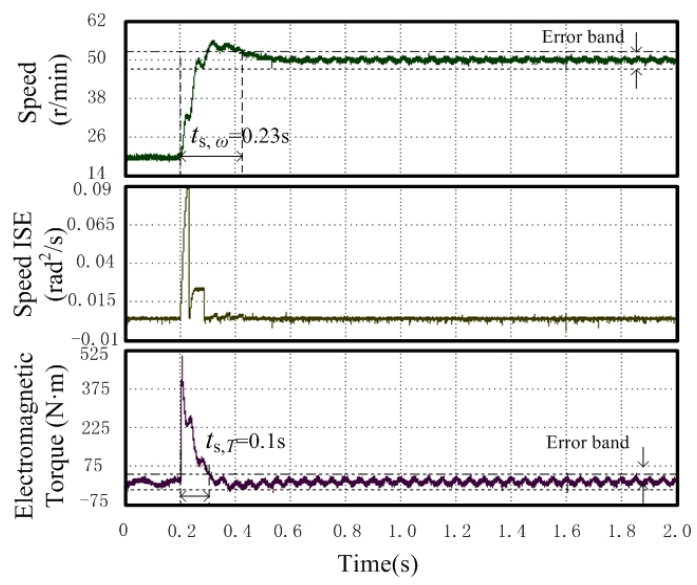

(b)

Fig. 16. Dynamic response curve using PI controller: (a) at load step from 0 to $140 \mathrm{~N} . \mathrm{m}$; (b) at speed step from 20 to $50 \mathrm{r} / \mathrm{min}$

parameter $\alpha$. Experimental results validate the effectiveness of the proposed algorithm.

\section{Acknowledgements}

This work was supported by the National Key Basic Research Program of China (973 Program) under Grant 2013CB035600, and the National Natural Science Foundation of China under Grant 51577134.

\section{References}

[1] Z. Q. Zhu, S. Ruangsinchaiwanich, and D. Howe, "Synthesis of cogging torque waveform from analysis 
of a single stator slot," IEEE Trans. Ind. Appl., vol. 42, no. 3, pp. 650-657, May-June 2006.

[2] C. L. Xia, J. X. Zhao, Y. Yan, and T. N. Shi, "A novel direct torque control of matrix converter-fed PMSM drives using duty cycle control for torque ripple reduction," IEEE Trans. Ind. Electron., vol. 61, no. 6, pp. 2700-2713, June 2014.

[3] S. T. Chen, C. Namuduri, and S. Mir, "Controllerinduced parasitic torque ripples in a PM synchronous motor," IEEE Trans. Ind. Appl., vol. 38, no. 5, pp. 1273-1281, October 2002.

[4] D. Zarko, D. Ban, and T. A. Lipo, "Analytical solution for cogging torque in surface permanent magnet motors using conformal mapping," IEEE Trans. Magn., vol. 44, no. 1, pp. 52-65, January 2008.

[5] P. S. Shin, H. Y. Kim and Y. B. Kim, "Minimization of Torque Ripple for an IPMSM with a Notched Rotor Using the Particle Swarm Optimization Method," JEET, vol. 9, no. 5, pp. 1577-1581, September 2014.

[6] T. Jahns, and W. Soong, "Pulsating torque minimization techniques for permanent magnet $\mathrm{AC}$ motor drives - a review," IEEE Trans. Ind. Electron., vol. 43, no. 2, pp. 321-330, April 1996.

[7] G. Ferretti, G. Magnani, and P. Rocco, "Modeling, identification, and compensation of pulsating torque in permanent magnet ac motors," IEEE Trans. Ind. Electron., vol. 45, no. 6, pp. 912-920, Dec. 1998.

[8] N. Vaks, D. Horvath, "Feedback-based mitigation of torque harmonics in interior permanent magnet synchronous machines," in IEMDC., pp. 564-570, May 2013.

[9] D. Flieller, N. K. Nguyen, etc., "A self-learning solution for torque ripple reduction for non-sinusoidal permanent magnet motor drives based on artificial neural networks," IEEE Trans. Ind. Electron., vol. 61, no. 61, pp. 655-666, Feb. 2014.

[10] M. Ruderman, A. Ruderman, and T. Bertram, "Observer-based compensation of additive periodic torque disturbances in permanent magnet motors," IEEE Trans. Ind. Informat., vol. 9, no. 2, pp. 11301138, May 2013.

[11] N. Matsui, T. Makino, and H. Satoh, "Autocompensation of torqueripple of direct drive motor by torque observer," IEEE Trans. Ind. Appl., vol. 29, no. 1, pp. 187-194, Jan/Feb 1993.

[12] Y. Abdel-Rady I. Mohamed, and E. F EI-Saadany, "A current control scheme with an adaptive internal model for torque ripple minimization and robust current regulation in PMSM drive systems," IEEE Trans. energy convers, vol. 23, no. 1, pp.92-100, March 2008.

[13] Y.A.I. Mohamed, “A newly designed instantaneoustorque control of direct-drive PMSM servo actuator with improved torque estimation and control characteristics," IEEE Trans. Ind. Electron., vol. 54, no. 5, pp. 2864-2873, Oct. 2007.
[14] G. Jayabaskaran, B. Adhavan, and V. Jagannathan, "Torque ripple reduction in permanent magnet synchronous motor driven by field oriented control using iterative learning control with space vector pulse width modulation," in ICCCI., Coimbatore, 2013.

[15] J. X. Xu, P. S. K, Y. J. Pan. T. Heng, and Lee Lam B. $\mathrm{H}$, "A modular control scheme for PMSM speed control with pulsating torque minimization," IEEE Trans. Ind. Electron., vol. 51, no. 3, pp. 526-536, June 2004.

[16] B. H. Lam, U. Nat, S. K. Panda, and J. X. Xu, "Reduction of periodic speed ripples in PM synchronous motors using iterative learning control," in IECON, vol. 2, pp. 1406-1411, 2000.

[17] K. C. Yeo, G. Heins and F. D. Boer, "Position Based Iterative Learning Control to minimise torque ripple for PMSMs," in IECON 2001, Melbourne, Australia, Nov. 2001

[18] Y. Yan, W. Li, W. Deng and G. Zhang, "Torque Ripple Minimization of PMSM Using PI Type Iterative Learning Control," in IECON 2014, Dallas, USA, Nov. 2014.

[19] K. Abidi and J. Xu, "Iterative Learning Control for Sampled-Data Systems: From Theory to Practice," IEEE Trans. Ind. Electron., vol. 58, no. 7, pp. 30023015, July 2011.

[20] W. Qian, S. K. Panda and J. Xu, "Torque Ripple Minimization in PM synchronous motors using iterative learning control," IEEE Trans. Power. Electron., vol. 19, no. 2, pp. 272-279, March. 2004.

[21] W. Z. Qian, S. K. Panda, and J. X. Xu, "Speed Ripple Minimization in PM synchronous motor using iterative learning control," IEEE Trans. energy convers, vol. 20, no. 1, pp. 53-61, March 2005.

[22] N. Amann, D. H. Owens, and E. rogers, "Iterative learning control using optimal feedback and feedforward actions," Internat. J. Control, vol. 65, pp. 277-293, July 1995.

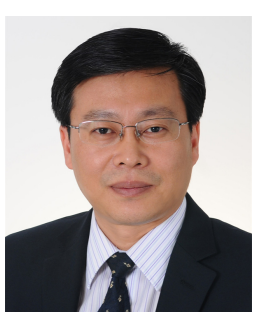

Changliang Xia He received the B.S. degree from Tianjin University, China, in 1990 and the M.S. and Ph.D. degrees from Zhejiang University, China, in 1993 and 1995 respectively, all in electrical engineering. He is currently a Professor at Tianjin University, and also Tianjin Polytechnic University. In 2008, he became a "Yangtze Fund Scholar" Distinguished Professor. He is currently supported by the National Science Fund for Distinguished Young Scholars. His research interests include electrical machines and their control systems, power electronics, and control of wind generators. 


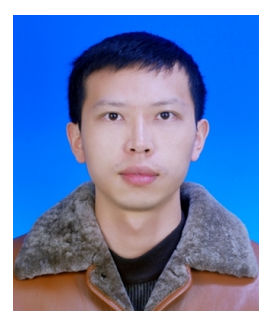

Weitao Deng He received B.S degree in electrical engineering from Tianjin University, Tianjin, in 2010 . He is currently working towards the Ph.D. in Tianjin University. His research interests include electrical machines, motor drives and power electronics.

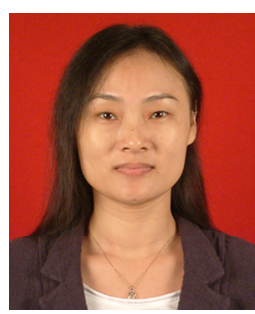

Tingna Shi She received the B.S. and M.S. degrees from Zhejiang University, China, in 1991 and 1996, respectively, and the Ph.D. degree from Tianjin University, China, in 2009. She is currently a Professor at Tianjin University. Her research interests include electrical machines and their control systems, power electronics, and electric drives

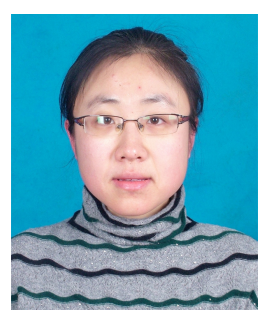

Yan Yan She received the B.S. and M.S. degrees from Tianjin University of Science and Technology, China, in 2004 and 2007 respectively, and the Ph.D. degree from Tianjin University, China, in 2010, all in electrical engineering. She is currently a lecturer at Tianjin University. Her research interests include the design and control of matrix converters for electrical drive applications and power converters for wind power generators. 